OPEN ACCESS

Edited by:

Jai Prakash

University of Twente, Netherlands

Reviewed by:

Pierpaolo Correale,

Unità di Oncologia Medica, Azienda

Ospedaliera

"Bianchi-Melacrino-Morelli", Italy

Antonio Rozzi,

Centre Hospitalier Régional Metz,

Thionville, France

*Correspondence:

Hou-Qun Ying

yinghouqun2013@163.com

Zhen Zong

zongzhenmd@126.com

†These authors have contributed equally to this work

Specialty section:

This article was submitted to

Cancer Molecular Targets and

Therapeutics,

a section of the journal

Frontiers in Oncology

Received: 08 April 2019

Accepted: 19 July 2019

Published: 14 August 2019

Citation:

You X-H, Wen C, Xia Z-J, Sun F, Li Y, Wang W, Fang Z, Chen $Q-G$, Zhang $L$,

Jiang $Y-H$, Wang $X-Z$, Ying $H-Q$ and Zong Z (2019) Primary Tumor

Sidedness Predicts Bevacizumab Benefit in Metastatic Colorectal Cancer Patients. Front. Oncol. 9:723.

doi: 10.3389/fonc. 2019.00723

\title{
Primary Tumor Sidedness Predicts Bevacizumab Benefit in Metastatic Colorectal Cancer Patients
}

\begin{abstract}
Xia-Hong You ${ }^{1 \dagger}$, Can Wen ${ }^{2 \dagger}$, Zi-Jin Xia ${ }^{3 \dagger}$, Fan Sun ${ }^{1}$, Yao $\mathrm{Li}^{3}$, Wei Wang ${ }^{3}$, Zhou Fang ${ }^{3}$, Qing-Gen Chen ${ }^{1}$, Lei Zhang ${ }^{1}$, Yu-Huang Jiang ${ }^{1}$, Xiao-Zhong Wang ${ }^{1}$, Hou-Qun Ying ${ }^{1,4 *}$ and Zhen Zong ${ }^{5 *}$
\end{abstract}

\begin{abstract}
${ }^{1}$ Department of Clinical Laboratory, Jiangxi Province Key Laboratory of Laboratory Medicine, The Second Affiliated Hospital of Nanchang University, Nanchang, China, ${ }^{2}$ Department of Clinical Laboratory, Jiangxi Cancer Hospital, Nanchang, China, ${ }^{3}$ Jiangxi Medical College, Nanchang University, Nanchang, China, ${ }^{4}$ Department of Nuclear Medicine, The Second Affiliated Hospital of Nanchang University, Nanchang, China, ${ }^{5}$ Department of Gastrointestinal Surgery, The Second Affiliated Hospital of Nanchang University, Nanchang, China
\end{abstract}

The emerging debate between primary tumor location and clinical outcome of bevacizumab treated metastatic colorectal cancer $(\mathrm{mCRC})$ continues. The aim of the present study is to investigate the association between the primary tumor location and clinical outcome of $115 \mathrm{mCRC}$ patients receiving bevacizumab based treatment. A meta-analysis including 21 studies was carried out to confirm the conclusion. In our prospective study, we found that right-sided mCRC commonly occurred in older cases $(p=0.03)$ with multiple-site metastasis $(p=0.03)$. Progression-free survival (PFS) of the left-sided patients undergoing bevacizumab plus a FOLFIRI regimen was superior to the right-sided cases $(p=0.03$, crude $\mathrm{HR}=0.31,95 \% \mathrm{Cl}=0.11-0.87$; adjusted $\mathrm{HR}=0.21$, $95 \% \mathrm{Cl}=0.06-0.66)$. The meta-analysis confirmed that efficacy of bevacizumab-based treatment in left-sided $\mathrm{mCRC}$ patients was better than the right-sided cases in the overall population $\left(P_{\mathrm{h}}=0.24\right.$, combined $\left.\mathrm{OR}=1.36,95 \% \mathrm{Cl}=1.07-1.72\right), \mathrm{RAS} / \mathrm{BRAF}$ wild-type $\left(P_{\mathrm{h}}=0.19\right.$, combined $\left.\mathrm{OR}=1.66,95 \% \mathrm{Cl}=1.17-2.34\right)$, clinical trial $\left(P_{\mathrm{h}}\right.$ $=0.23$, combined $\mathrm{OR}=1.42,95 \% \mathrm{Cl}=1.07-1.88)$, Caucasian population $\left(P_{\mathrm{h}}=\right.$ 0.18 , combined $\mathrm{OR}=1.37,95 \% \mathrm{Cl}=1.02-1.85)$ and first-line $\left(P_{\mathrm{h}}=0.19\right.$, combined $\mathrm{OR}=1.48,95 \% \mathrm{Cl}=1.13-1.96)$ subgroups. Improved survival of bevacizumab plus chemotherapy treated left-sided $\mathrm{mCRC}$ patients was observed in the overall population $\left[P_{\mathrm{h}}<0.01\right.$, combined MSR $=1.09,95 \% \mathrm{Cl}=1.00-1.18$ for PFS; $P_{\mathrm{h}}<0.01$, combined $\mathrm{MSR}=1.24,95 \% \mathrm{Cl}=1.13-1.36$ for overall survival $(\mathrm{OS})]$, especially in the RAS/BRAF wild-type $\left(P_{\mathrm{h}}=0.09\right.$, combined $\mathrm{MSR}=1.10,95 \% \mathrm{Cl}=1.03-1.19$ for PFS; $P_{\mathrm{h}}=0.02$, combined MSR $=1.34,95 \% \mathrm{Cl}=1.21-1.49$ for OS). These findings indicate that primary tumor sidedness can predict clinical outcome of bevacizumab-treated RAS/BRAF wild-type $\mathrm{mCRC}$ patients and the left-sided patients may benefit more from bevacizumab plus FOLFIRI.

Keywords: primary tumor sidedness, bevacizumab, mCRC, prognosis, survival 


\section{INTRODUCTION}

Colorectal cancer (CRC) is the fourth most commonly diagnosed malignancy and the second leading cause of cancer-related death worldwide (1). Due to the invasiveness of digestive endoscopy and limited sensitivity of fecal immunochemical tests, the majority of new cases are usually diagnosed at the advanced stages of the disease (2). In addition to palliative surgery and radiochemotherapy, anti-epidermal growth factor receptor monoclonal antibody (anti-EGFR $\mathrm{mAb}$ ), and antivascular endothelial growth factor (anti-VEGF) $\mathrm{mAb}$ have been used to prolong the survival of metastatic CRC (mCRC) patients (3). Nevertheless, clinical outcomes of the two inhibitor managed mCRC patients remain unsatisfactory in the clinic, with evidence showing that objective response rates (ORRs) and median progression-free survival (PFS) of cetuximab or bevacizumab-based chemotherapy are 59.6\% and 10.5 months in KRAS-wild patients, and $62.1 \%$ and 9.5 months in the overall patient population $(4,5)$, respectively. Thus, robust prognostic and predictive factors which can more precisely stratify suitable patients to receive the optimal biological therapy may help to improve the clinical efficacy and outcome.

Recently, accumulating evidence has shown the significant differences in clinical characteristics, anatomic structure, embryological origin, and the genetic mutation profile between left- and right-sided CRC (6). The role of primary tumor localization has extensively increased attention, for its impact on response to biological therapy and the survival of the patient (7-11). The latest clinical trials and meta-analyses confirmed that KRAS-wild patients with left-sided mCRC derived great benefit from EGFR-inhibitor contained treatment $(12,13)$, and the inhibitor has been recommended as a first-line therapeutic treatment for patients in the 2017 National Comprehensive Cancer Network guideline (14).

Nowadays, several studies reported the involvement of primary tumor sidedness in clinical efficacy and prognosis of refractory mCRC individuals with treatment of bevacizumabcontaining chemotherapy (15-17). Nevertheless, no consensus of the association between them has been achieved and its controversy still continues (18). FIRE-3, AVF2107g, and NO16966 trials show that the efficacy of bevacizumab is independent of primary tumor sidedness in mCRC patients (13, 19). On the contrary, other trials and retrospective studies imply a significantly different survival in two-sided patients undergoing bevacizumab-based therapy $(15-17,20)$. Studies performed by Aljehani and Boisen et al. reported that two-sided patients could benefit from bevacizumab and chemotherapy, whereas a rightsided cancer origin was associated with poor response and high mortality among patients undergoing bevacizumab, compared to left-sided cases $(7,20)$.

In the present study, a prospective study including 115 bevacizumab-treated mCRC patients and a meta-analysis containing 13 clinical trials and eight non-clinical trials was carried out to comprehensively understand the role of primary tumor location in the effectiveness of bevacizumab in mCRC patients.

\section{MATERIALS AND METHODS}

\section{Eligible Population}

To investigate the involvement of primary tumor sidedness in the prognosis of bevacizumab treated mCRC patients, we prospectively screened eligible mCRC patients at the Second Hospital of Nanchang University and Jiangxi Cancer Hospital from August 2012 to August of 2015. The inclusion and exclusion criteria are as follows: (1) all of the included patients were first confirmed as mCRC through both imaging and pathological examination; (2) all enrolled patients received bevacizumab and standard chemotherapy; (3) all eligible cases were willing to participate in the study and written informed consent was obtained from all enrolled patients. Those without definite diagnosis or bevacizumab-based therapy were excluded from the study. Tumors located at the caecum to the transverse colon were defined as right-sided CRC, and those located within the splenic flexure, and beyond were considered as left-sided. The present study was approved by the Medical Ethics Committees of the Second Affiliated Hospital of Nanchang University and Jiangxi Cancer Hospital, respectively.

\section{Follow-Up and Clinical Response Evaluation}

We performed follow-ups each 3 months in the first 2 years, and each 6 months in the third year to achieve (PFS) and overall survival (OS), with a deadline of August 2018. The time since the enrolment day to tumor enlargement or new metastases and death or its deadline were defined as PFS and OS, respectively. During the same time, clinical efficacy of bevacizumab and adjuvant chemotherapy was assessed after 3 months of regimen usage according to the response evaluation criteria in solid tumors (RECIST) guideline (version 1.1). The evaluated responses were defined as complete response (CR), partial response (PR), stable disease (SD), and progressive disease $(\mathrm{PD})$, respectively. We calculated objective response rate (ORR) according to the evaluated result.

\section{Relevant Study Identification and Data Extraction}

In order to further understand the association between primary tumor location and bevacizumab efficacy in mCRC patients, we screened and identified eligible studies to perform a meta-analysis. A comprehensive retrieval was carried out by two investigators (X-HY and Z-JX) in PUBMED, EMBASE, and the Cochrane Library as well as the CNKI database until June 2018. The following medical search terms were selected to screen relative articles: "rectal, colon, colorectal," "cancer, tumor, neoplasms, or carcinoma," "sided, sidedness, side, location, localization, site," and "prognosis, survival, outcome." Moreover, we manually searched for additional studies by screening the references of the relevant articles, and enrolled eligible studies according to the following inclusion criteria: (1) original article reported the survival of left- and rightsided mCRC with treatment of bevacizumab and chemotherapy; (2) relevant study provided clinical characteristics, clinical response, median survival time or hazard ratio (HR) and $95 \%$ 
TABLE 1 | The baseline characteristics of $115 \mathrm{mCRC}$ patients in the present study.

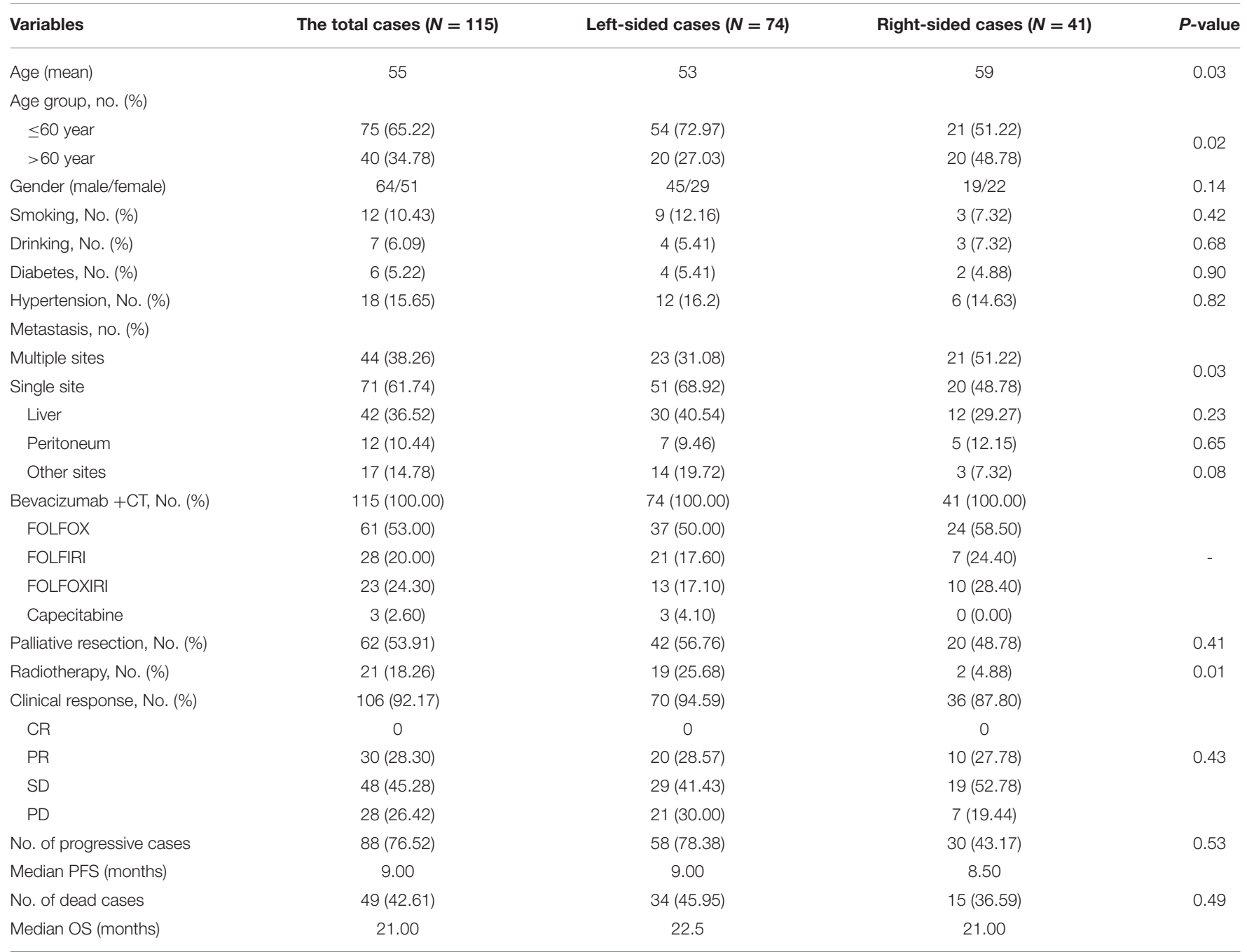

$C T$, chemotherapy; $C R$, complete response; PR, partial response; SD, stable disease; PD, progressive disease; PFS, progression-free survival; OS, overall survival; FOLFOX, fluorouracil, leucovorin, and oxaliplatin; FOLFIRI, fluorouracil, leucovorin, and irinotecan; FOLFOXIRI, oxaliplatin, fluorouracil, and irinotecan.
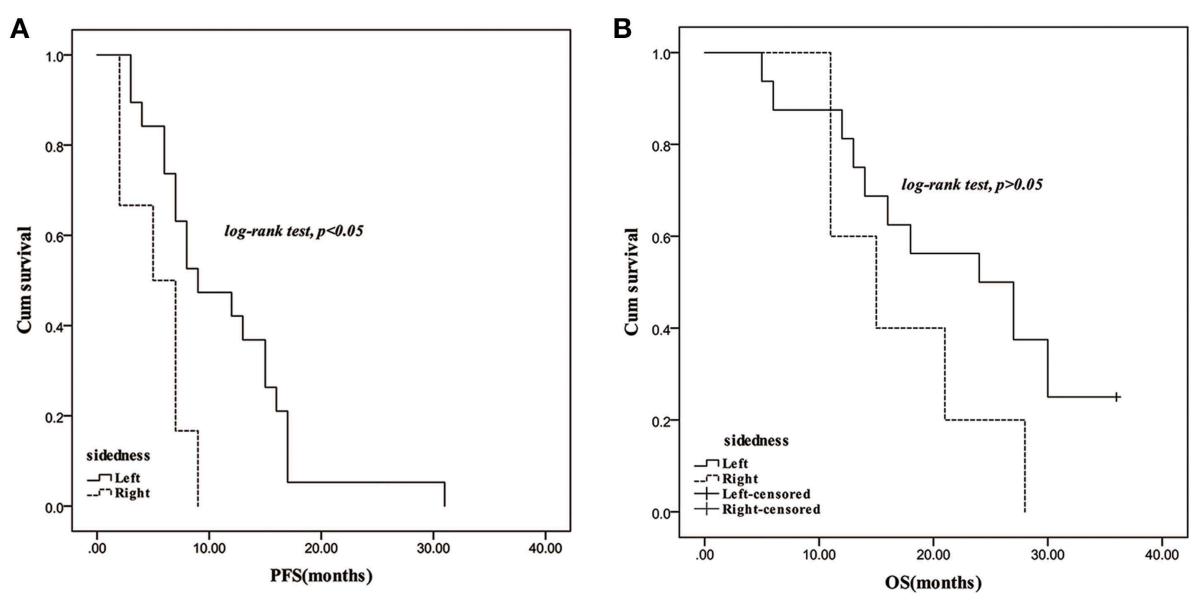

FIGURE 1 | Survival comparison between right- and left-sided mCRC patients with treatment of bevacizumab plus FOLFIRI regimen. (A): PFS; (B): OS. 
confidential interval (CI). Subsequently, two investigators (XHY and Z-JX) independently extracted clinical characteristics (first author, publication year, region, race, study design, clinical trial, treatment, included patients, median age, gender), response and survival data. Inconsistent data was discussed with a third investigator $(\mathrm{CW})$ to reach a consensus by analyzing the full-text.

\section{Statistics}

The baseline characteristics of the included patients and the response data were presented by numbers and proportions. PFS and OS emerged as the median survival in months. The relationship between primary tumor sidedness and clinical response to bevacizumab was assessed by Pearson $\chi^{2}$ test, and odds ratio (OR) and 95\%CI were selected to measure the strength between them. Kaplan-Meier curve (log-rank test) and Cox regression analysis were selected to examine survival difference between left- and right-sided mCRC cases. HR and median survival ratio (MSR) were presented to show the strength between them. Heterogeneity of eligible studies in the metaanalysis was evaluated by Q test and estimated $I^{2}, P_{\mathrm{h}}<0.1$ or $I^{2}>50 \%$ was recognized as a significant heterogeneity between them. According to the heterogeneity test, the $\mathrm{Z}$ test in the fixed $\left(P_{\mathrm{h}}>0.1\right)$ or random $\left(P_{\mathrm{h}}<0.1\right)$ model was selected to analyze the combined effect in the meta-analysis. All of the statistics were performed using the SPSS statistical 17.0 (SPSS Inc., Chicago, IL) and Stata 11.0 software (Stata Corporation, College Station, TX), $p<0.05$ was recognized as a statistical significance between the comparison.

\section{RESULTS}

In the present study, 74 left-sided mCRC patients and 41 rightsided cases were enrolled according to inclusion and exclusion criteria. The baseline characteristics are described in Table 1. The left-sided patients were significantly younger than the rightsided cases $(p=0.03)$, multiple sites metastasis $(p=0.03)$ was frequently observed in right-sided patients compared to the leftsided individuals. In addition to all of the patients that received bevacizumab and chemotherapy, 56.76\% of the left-sided mCRC patients and $48.78 \%$ of the right-sided patients received palliative resection, and radiotherapy-treated left-sided cases were higher than the right-sided patient $(p=0.01)$. Due to intolerance of chemotherapy cytotoxicity effect, nine patients retired from the study and the response and survival data was only obtained from the remaining 106 cases. Among them, 30, 48, and $28 \mathrm{mCRC}$ cases were evaluated as PR, SD, and PD, respectively. Disease

TABLE 2 | Baseline characteristics of included studies.

\begin{tabular}{|c|c|c|c|c|c|c|c|c|c|c|}
\hline Study & Population & Clinical trial & $\begin{array}{l}\text { Treatment } \\
\text { line }\end{array}$ & $\begin{array}{l}\text { RAS/BRAF } \\
\text { status }\end{array}$ & Therapeutic regimen & Cases & $\begin{array}{l}\text { Left- } \\
\text { side }\end{array}$ & $\begin{array}{l}\text { Right- } \\
\text { side }\end{array}$ & $\begin{array}{l}\text { Male/ } \\
\text { Female }\end{array}$ & Outcome \\
\hline Calvetti et al. (21) & Caucasian & Non-clinical trial & First line & Wild type & Chemotherapy + Bev & 81 & NA & NA & NA & OS \\
\hline Tejpar et al. (13) & Caucasian & FIRE-3 & First line & Wild type & Chemotherapy + Bev & 199 & 149 & 50 & NA & OS, PFS, ORR \\
\hline Lu et al. (22) & Asian & Non-clinical trial & First line & Wild type & Chemotherapy + Bev & 54 & 30 & 24 & $37 / 17$ & OS, PFS, ORR \\
\hline He et al. (23) & Asian & Non-clinical trial & First line & Unknown & Chemotherapy + Bev & 164 & 86 & 78 & $100 / 64$ & OS \\
\hline Arnold et al. (12) & Caucasian & PEAK & First line & Wild type & Chemotherapy + Bev & 68 & 54 & 14 & NA & OS, PFS, ORR \\
\hline Sun et al. (24) & Asian & Non-clinical trial & $\begin{array}{l}\text { Non-first } \\
\text { line }\end{array}$ & Unknown & Chemotherapy + Bev & 217 & 138 & 79 & $120 / 97$ & OS, PFS, ORR \\
\hline Houts et al. (25) & Mix & CALGB 80405 & First line & Wild type & Chemotherapy + Bev & 241 & 162 & 79 & $140 / 114$ & OS \\
\hline Arnold et al. (12) & Caucasian & CALGB 80405 & First line & Wild type & Chemotherapy + Bev & 230 & 152 & 78 & NA & PFS, ORR \\
\hline Bazarbashi et al. (26) & Asian & NCT01311050 & First line & Unknown & Chemotherapy + Bev & 53 & 42 & 11 & $28 / 25$ & OS, PFS, ORR \\
\hline Ulivi et al. (27) & Caucasian & NCT01878422 & First line & Unknown & Chemotherapy + Bev & 53 & 30 & 23 & NA & OS, PFS \\
\hline Arora et al. (28) & Caucasian & $\begin{array}{l}\text { Phase } 1 \text { clinical } \\
\text { trial }\end{array}$ & $\begin{array}{l}\text { Non-first } \\
\text { line }\end{array}$ & Unknown & Chemotherapy + Bev & 121 & 86 & 35 & $85 / 36$ & OS, PFS \\
\hline Demircan et al. (29) & Asian & Non-clinical trial & First line & Unknown & Chemotherapy + Bev & 360 & NA & NA & $201 / 159$ & OS, PFS \\
\hline Reinacher et al. (30) & Caucasian & AIO KRK 0207 & First line & Unknown & Chemotherapy + Bev & 414 & NA & NA & NA & OS, PFS \\
\hline Artaç et al. (31) & Asian & Non-clinical trial & First line & $\begin{array}{l}\text { Wild type, } \\
\text { Mutant type }\end{array}$ & Chemotherapy + Bev & 371 & 270 & 101 & 228/335 & OS, PFS \\
\hline Loupakis et al. (32) & Mix & AVF2107g & First line & Unknown & Chemotherapy + Bev & 298 & 195 & 103 & NA & OS, PFS \\
\hline Loupakis et al. (32) & Mix & N016966 & First line & Unknown & Chemotherapy + Bev & 497 & 380 & 117 & NA & OS, PFS \\
\hline Cremolini et al. (15) & Caucasian & TRIBE & First line & $\begin{array}{l}\text { Wild type, } \\
\text { Mutant type }\end{array}$ & Chemotherapy + Bev & 358 & 242 & 116 & $218 / 140$ & OS, PFS, ORR \\
\hline Satake et al. (33) & Asian & JACCRO CC-11 & First line & Mutant type & Chemotherapy + Bev & 62 & 45 & 17 & $34 / 28$ & PFS, ORR \\
\hline Chibaudel et al. (34) & Caucasian & DREAM & $\begin{array}{l}\text { Non-first } \\
\text { line }\end{array}$ & $\begin{array}{l}\text { Wild type, } \\
\text { Mutant type }\end{array}$ & Chemotherapy + Bev & 348 & 250 & 98 & NA & OS \\
\hline Nakamura et al. (35) & Asian & Non-clinical trial & First line & Unknown & Chemotherapy + Bev & 112 & NA & NA & NA & OS \\
\hline You et al. & Asian & Non-clinical trial & $\begin{array}{l}\text { Non-first } \\
\text { line }\end{array}$ & Unknown & Chemotherapy + Bev & 115 & 74 & 41 & $64 / 51$ & OS, PFS, ORR \\
\hline
\end{tabular}

Bev, bevacizumab; OS, overall survival; PFS, progression-free survival; ORR, objective response rate; NA, not available. 
A

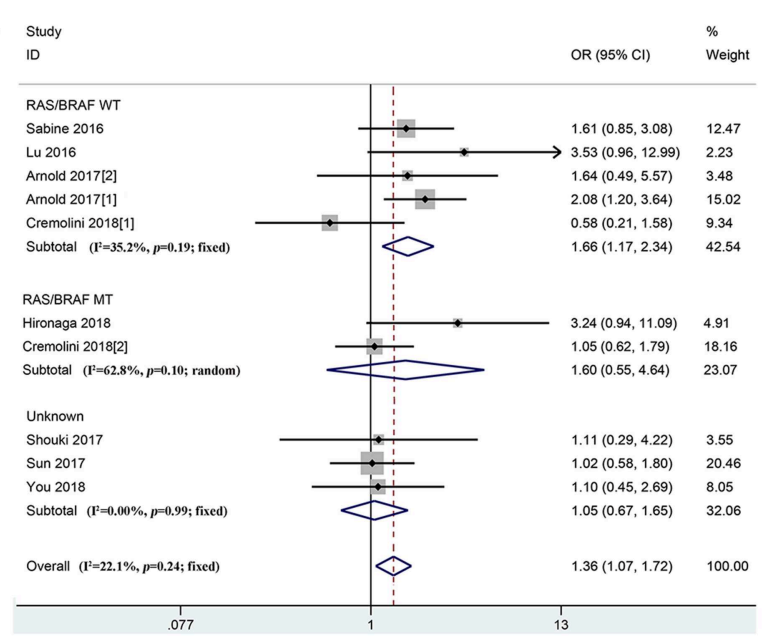

C

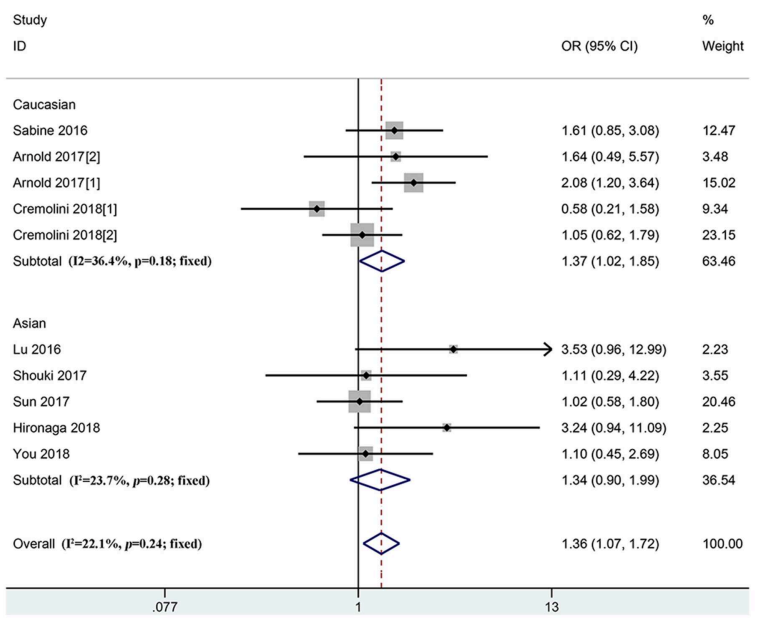

B

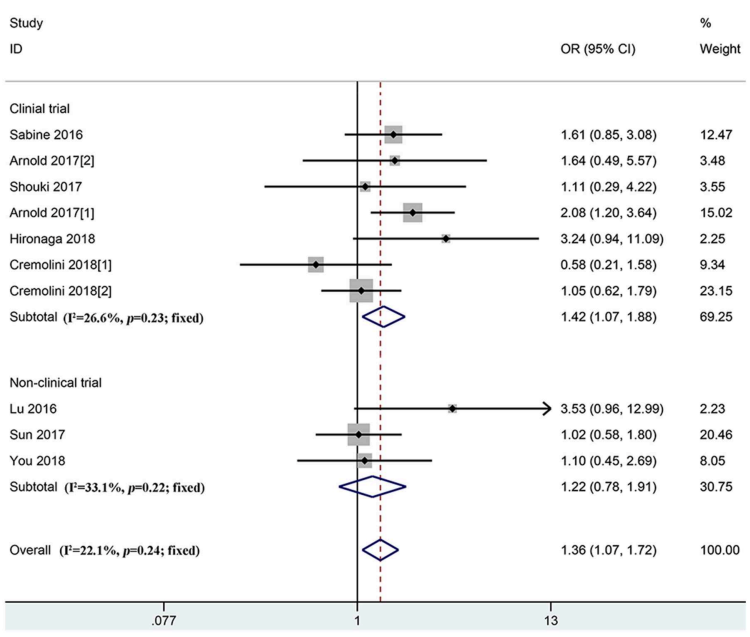

D

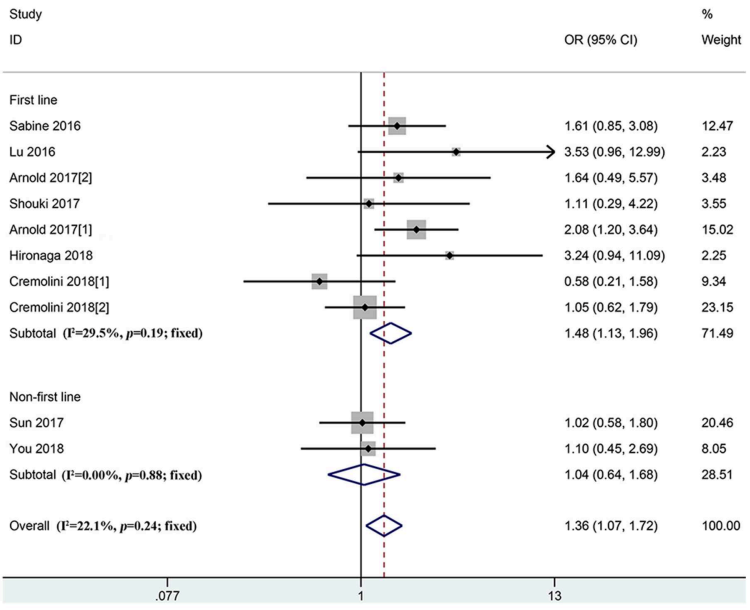

FIGURE 2 | Combined effect of objective response rates (left vs. right) in the overall population and subgroup, stratified by RAS/BRAF status (A), study design (B), population (C), treatment-line (D).

progression was observed in 88 patients and 49 patients died, with a median PFS and OS of 9 and 21 months, respectively.

In response to bevacizumab, $28.57 \%$ of left-sided mCRC patients, and $27.78 \%$ of the right-sided cases were assessed as $\mathrm{CR} / \mathrm{PR}$, respectively. No difference of ORR was observed between the right- and left-sided cases and the two-sided patients stratified by different therapeutic regimen (Supplementary Table 1). In the follow-up period, disease progression was observed in 78.38 and $73.17 \%$ of the left- and right-sided cases, and the median PFS had no difference between them ( 9 vs. 8.5 months). Thirty-four left-sided patients and 15 right-sided individuals died, and the two-sided patients harbored 22.5 and 21 months of median OS, respectively. No significant PFS and OS difference was observed between the two-sided overall patients. According to the therapeutic regimen, there was no significant survival difference between the two-sided cases regardless of palliative surgery or radiotherapy. No survival difference was observed between the two-sided patients undergoing bevacizumab combined FOLFOX or FOLFOXIRI regimens. However, PFS of right-sided patients undergoing bevacizumab and a FOLFIRI regimen was significantly inferior to the left-sided cases $(p=0.03$, crude HR $=$ $0.31,95 \% \mathrm{CI}=0.11-0.87 ; p=0.01$, adjusted $\mathrm{HR}=0.21,95 \% \mathrm{CI}$ $=0.06-0.66)$ (Figure 1 and Supplementary Tables 2, 3).

In accordance with the inclusion criteria of eligible studies, a total of 21 studies including 4,416 patients were enrolled in the meta-analysis (12, 13, 15, 21-35) (Supplementary Figure 1). Among them, two prospective and 19 retrospective studies were included. Seventeen (11 clinical trials and 6 nonclinical trials) and four studies reported the first-line and non-first-line usage of bevacizumab and chemotherapy in mCRC cases, respectively. Moreover, 9, 16, and 19 eligible studies reported clinical efficacy of the therapeutic regimen, PFS and OS of the patients, respectively. The baseline characteristics of included studies are described in Table 2. Combined ORR of left-sided mCRC patients was superior to right-sided cases $\left(P_{\mathrm{h}}=0.24\right.$, combined $\mathrm{OR}=1.36,95 \% \mathrm{CI}$ $=1.07-1.72$ ) (Figure 2 and Supplementary Table 4). When stratifying according to the $R A S / B R A F$ status, population, study 

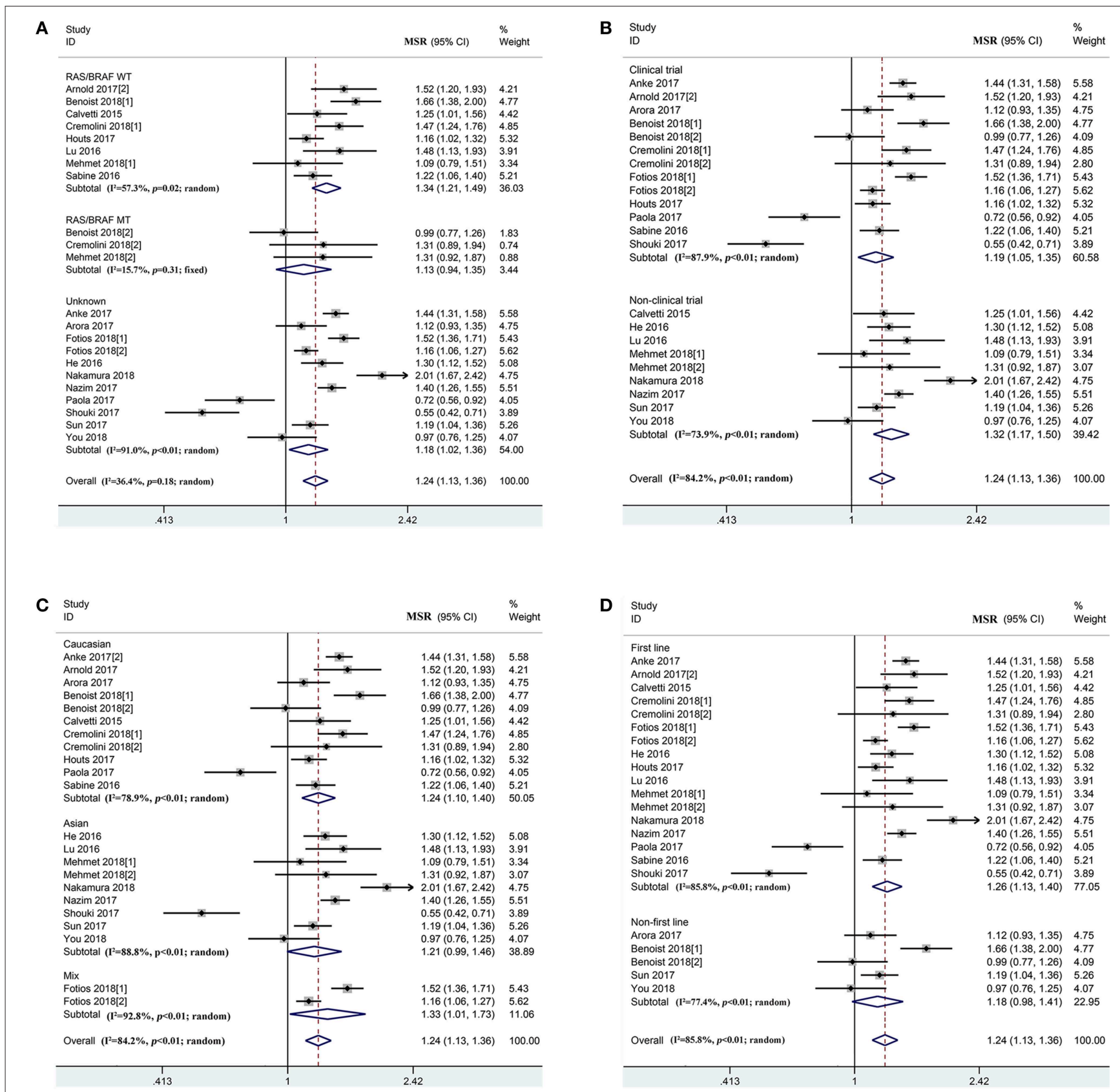

FIGURE 3 | Combined effect of median overall survival ratio (left vs. right) in the overall population and subgroup, stratified by RAS/BRAF status (A), study design (B), population (C), treatment-line (D).

design and treatment line, we found that primary tumor sidedness was significantly associated with clinical response to bevacizumab and chemotherapy in the RAS/BRAF wildtype patients $\left(P_{\mathrm{h}}=0.19\right.$, combined $\mathrm{OR}=1.66,95 \% \mathrm{CI}=$ $1.17-2.34)$ (Figure 2A), clinical trials $\left(P_{\mathrm{h}}=0.23\right.$, combined $\mathrm{OR}=1.42,95 \% \mathrm{CI}=1.07-1.88)$ (Figure 2B), Caucasian population $\left(P_{\mathrm{h}}=0.18\right.$, combined $\mathrm{OR}=1.37,95 \% \mathrm{CI}=$ 1.02-1.85) (Figure 2C), as well as first-line $\left(P_{\mathrm{h}}=0.19\right.$, combined $\mathrm{OR}=1.48,95 \% \mathrm{CI}=1.13-1.96) \quad($ Figure 2D) subgroup, respectively.
According to the prognosis of mCRC patients in the overall population, PFS $\left(P_{\mathrm{h}}<0.01\right.$, combined MSR $=1.09,95 \% \mathrm{CI}=$ $1.00-1.18)$ and $\mathrm{OS}\left(P_{\mathrm{h}}<0.01\right.$, combined MSR $=1.24,95 \% \mathrm{CI}$ $=1.13-1.36)$ within left-sided mCRC patients were significantly longer than those of the right-sided cases (Figure 3 and Supplementary Figure 2, Supplementary Table 5). Moreover, compared to right-sided mCRC patients, bevacizumabtreated left-sided mCRC cases showed improved PFS in the $R A S / B R A F$ wild-type $\left(P_{\mathrm{h}}=0.09\right.$, combined $\mathrm{MSR}=1.10$, $95 \% \mathrm{CI}=1.03-1.19)$ (Supplementary Figure 2A), non-clinical 
trials $\left(P_{\mathrm{h}}=0.12\right.$, combined MSR $=1.23$, 95\%CI $=1.14-$ 1.32) (Supplementary Figure 2B), and mixed population $\left(P_{\mathrm{h}}=0.17\right.$, combined $\left.\mathrm{MSR}=1.15,95 \% \mathrm{CI}=1.08-1.23\right)$ (Supplementary Figure 2C) subgroups. In addition, primary tumor sidedness was significantly associated with improved OS in $\mathrm{mCRC}$ patients undergoing bevacizumab and chemotherapy regardless of the study design (Figure 3B), population (Figure 3C), and treatment line (Figure 3D), especially in $R A S / B R A F$ wild-type patients $\left(P_{\mathrm{h}}=0.02\right.$, combined MSR $=$ $1.34,95 \% \mathrm{CI}=1.21-1.49)($ Figure 3A$)$.

\section{DISCUSSION}

The impact of primary tumor location on bevacizumab plus adjuvant chemotherapy in mCRC patients remains controversial $(16,18)$. In this study, we found that left-sided mCRC patients could benefit more from bevacizumab plus FOLFIRI compared with its counterpart. With the large sample size, the robust results of the meta-analysis showed that clinical efficacy and survival of bevacizumab treated left-sided patients was significantly superior to right-sided patients.

1. Advanced CRC is a heterogeneous disease with a varied clinical efficacy and prognosis. Left- and right-sided diseases are reported to be distinct in clinical characteristics and mutation profiles of oncogenes and anti-oncogenes as well as clinical outcomes (36-38). Thus, common therapeutic strategies such as anti-VEGF and anti-EGFR antibody, essential pathway kinase and immune checkpoint inhibitors should be carefully selected based on the patient $(39,40)$. According to bevacizumab, the controversy is still undergoing with which kind of patients should to use suitably. Loupakis et al. reported that clinical outcomes of both two-sided mCRC patients were improved by treatment with bevacizumab and chemotherapy (41). In our study, we found that right-sided mCRC commonly occurred in older patients with multiple site metastasis, which is consistent with the report by Yang et al. (42). Our previous study indicated that prognosis of chemotherapy-treated right-sided mCRC patients was inferior to left-sided cases (43). Our prospective study showed that PFS of bevacizumab and FOLFIRI treated left-sided patients was significantly longer than the right-sided cases. Moreover, the meta-analysis indicated that the effect of bevacizumabbased treatment in left-sided mCRC patients was better than that of right-sided cases in the RAS/BRAF wild-type, clinical trial, Caucasian population, and first-line subgroups. It demonstrated that primary tumor sidedness could predict clinical efficacy and survival of mCRC patients with treatment of bevacizumab and chemotherapy, and the left-sided patients could benefit from a longer survival time from the therapeutic regimen than the right-sided cases, especially in bevacizumab, and FOLFIRI treated patients.

As we know, bevacizumab can combine with VEGF to inhibit angiogenesis. Compared to the proximal colon, VEGF was observed to be abundantly expressed in CRC in the distal colon and rectum $(41,44,45)$. Moreover, chromosomal instability (CIN) was commonly observed in $\sim 75 \%$ of leftsided patients and the right-sided cases usually harbored high microsatellite instability (MSI), a CpG island methylator phenotype (CIMP) as well as a BRAF mutation (46). The outcome of consensus molecular subtype (CMS) 2/4 mCRC patients, with intermediate-to-high CIN, was obviously improved following bevacizumab and chemotherapy, the CMS $1 / 3$ patients with unstable MSI and elevated CIMP, as well as low CIN could not derive further benefits from the inhibitor (47-49). In addition, distinctive gut microbiome features were observed to vary depending on primary tumor location of CRC $(6,50,51)$. The gut microbiome could modulate the response to anti-PD-1 immunotherapy and adjuvant chemotherapy in melanoma and CRC (52, 53). Microbiota has been linked to chronic inflammation. Severe inflammation was reported to associated with a poor response to bevacizumab (53) and significantly higher fibrinogen to pre-albumin ratio was detected in right-sided mCRC cases compared to its counterpart (43). The above causes may therefore help us better understand the effect and prognosis of primary tumor location in bevacizumab and chemotherapy treated mCRC patients.

To the best of our knowledge, the present study is the first to perform this meta-analysis with the largest sample size to date, to investigate the prognostic, and predictive role of primary tumor location in bevacizumab-treated mCRC patients. However, the following limitations should be addressed to understand the results of our study. First, the prospective-study design used in the present study was a small sample size, including patients from only two hospitals within the same region. This might restrict a robust conclusion in our study. Second, the KRAS/BRAF mutation was not detected and we did not investigate the impact of it on bevacizumab efficacy and the survival of mCRC patients.

In summary, our findings illustrate that the clinical outcome of bevacizumab treated left-sided mCRC patients is superior to right-sided patients, particularly in the wild-type $R A S / B R A F$ subgroup and bevacizumab and FOLFIR1 treated patients. Primary tumor sidedness is an effective factor used to predict the clinical response to bevacizumab and the prognosis of the patient. Considering the limitations of our study, randomized controlled trials from multiple-regions with large sample sizes are needed to verify our results.

\section{DATA AVAILABILITY}

All datasets generated for this study are included in the manuscript and/or the Supplementary Files.

\section{ETHICS STATEMENT}

Written informed consent was obtained from each enrolled patient, and the present study was approved by Medical Ethnic Committees of the Second Affiliated Hospital of Nanchang University and Jiangxi Cancer Hospital, respectively. 


\section{AUTHOR CONTRIBUTIONS}

$\mathrm{X}-\mathrm{HY}$ selected the eligible sample in the first section, screened, and selected the eligible study in the meta-analysis and performed all the statistics. CW provided the sample resource, selected the eligible patients, and prepared the clinical characteristics of each included patient in the first section. Z-JX contributed to screen, select, and identify the eligible study, prepared the clinical and survival data, and performed the statistics in the meta-analysis. FS, YL, and WW contributed to follow-up and characteristics acquisition in the first section. ZF, Q-GC, and LZ contributed to clinical and survival data acquisition in the second section. Y-HJ contributed to check-up the data. X-ZW, H-QY, and ZZ provided the idea, established the study design, revised, and approved the manuscript.

\section{REFERENCES}

1. Bray F, Ferlay J, Soerjomataram I, Siegel RL, Torre LA, Jemal A. Global cancer statistics 2018: GLOBOCAN estimates of incidence and mortality worldwide for 36 cancers in 185 countries. CA Cancer J Clin. (2018) 68:394424. doi: $10.3322 /$ caac. 21492

2. Strum WB. Colorectal adenomas. N Engl J Med. (2016) 374:106575. doi: 10.1056/NEJMra1513581

3. Benson AB III, Venook AP, Cederquist L, Chan E, Chen YJ, Cooper HS, et al. Colon cancer, version 1.2017, NCCN clinical practice guidelines in oncology. J Natl Compr Canc Netw. (2017) 15:370-98. doi: 10.6004/jnccn.2017.0036

4. Hurwitz HI, Tan BR, Reeves JA, Xiong H, Somer B, Lenz HJ, et al. Phase II randomized trial of sequential or concurrent FOLFOXIRI-bevacizumab versus FOLFOX-bevacizumab for metastatic colorectal cancer (STEAM). Oncologist. (2018) 24:921-32. doi: 10.1634/theoncologist.2018-0344

5. Venook AP, Niedzwiecki D, Lenz HJ, Innocenti F, Fruth B, Meyerhardt JA, et al. Effect of first-line chemotherapy combined with cetuximab or bevacizumab on overall survival in patients with KRAS wild-type advanced or metastatic colorectal cancer: a randomized clinical trial. JAMA. (2017) 317:2392-401. doi: 10.1001/jama.2017.7105

6. Stintzing S, Tejpar S, Gibbs P, Thiebach L, Lenz HJ. Understanding the role of primary tumour localisation in colorectal cancer treatment and outcomes. Eur J Cancer. (2017) 84:69-80. doi: 10.1016/j.ejca.2017.07.016

7. Boisen MK, Johansen JS, Dehlendorff C, Larsen JS, Osterlind K, Hansen $J$, et al. Primary tumor location and bevacizumab effectiveness in patients with metastatic colorectal cancer. Ann Oncol. (2013) 24:25549. doi: $10.1093 /$ annonc/mdt253

8. Passardi A, Nanni O, Tassinari D, Turci D, Cavanna L, Fontana A, et al. Effectiveness of bevacizumab added to standard chemotherapy in metastatic colorectal cancer: final results for first-line treatment from the ITACa randomized clinical trial. Ann Oncol. (2015) 26:12017. doi: 10.1093/annonc/mdv130

9. Sunakawa Y, Ichikawa W, Tsuji A, Denda T, Segawa Y, Negoro Y, et al. Prognostic impact of primary tumor location on clinical outcomes of metastatic colorectal cancer treated with cetuximab plus oxaliplatin-based chemotherapy: a subgroup analysis of the JACCRO CC-05/06 trials. Clin Colorectal Cancer. (2017) 16:e171-80. doi: 10.1016/j.clcc.2016.09.010

10. Boeckx N, Koukakis R, Op de Beeck K, Rolfo C, Van Camp G, Siena $\mathrm{S}$, et al. Primary tumor sidedness has an impact on prognosis and treatment outcome in metastatic colorectal cancer: results from two randomized first-line panitumumab studies. Ann Oncol. (2017) 28:18628. doi: 10.1093/annonc/mdx119

11. Boeckx N, Koukakis R, Op de Beeck K, Rolfo C, Van Camp G, Siena $S$, et al. Effect of primary tumor location on second- or later-line treatment outcomes in patients with RAS wild-type metastatic colorectal cancer and all treatment lines in patients with RAS mutations in four

\section{ACKNOWLEDGMENTS}

This report was supported by the National Natural Science Foundation of China (Grant Number: 81702090), the Natural Science Youth Foundation of Jiangxi Province (Grant Numbers: 20171BAB215054, 81860433), and the Key Technology Research and Development Program of Jiangxi Province (Grant Number: 20171BBG70049).

\section{SUPPLEMENTARY MATERIAL}

The Supplementary Material for this article can be found online at: https://www.frontiersin.org/articles/10.3389/fonc. 2019.00723/full\#supplementary-material

randomized panitumumab studies. Clin Colorectal Cancer. (2018) 17:170-8 e3. doi: 10.1016/j.clcc.2018.03.005

12. Arnold D, Lueza B, Douillard JY, Peeters M, Lenz HJ, Venook A, et al. Prognostic and predictive value of primary tumour side in patients with RAS wild-type metastatic colorectal cancer treated with chemotherapy and EGFR directed antibodies in six randomized trials. Ann Oncol. (2017) 28:171329. doi: 10.1093/annonc/mdx175

13. Tejpar S, Stintzing S, Ciardiello F, Tabernero J, Van Cutsem E, Beier F, et al. Prognostic and predictive relevance of primary tumor location in patients with RAS wild-type metastatic colorectal cancer: retrospective analyses of the CRYSTAL and FIRE-3 trials. JAMA Oncol. (2016) 3:194201. doi: 10.1001/jamaoncol.2016.3797

14. Chen G. [Interpretation of the updates of NCCN 2017 version 1.0 guideline for colorectal cancer]. Zhonghua Wei Chang Wai Ke Za Zhi. (2017) 20:28-33. doi: 10.3760/cma.j.issn.1671-0274.2017.01.007

15. Cremolini C, Antoniotti C, Lonardi S, Bergamo F, Cortesi E, Tomasello $\mathrm{G}$, et al. Primary tumor sidedness and benefit from FOLFOXIRI plus bevacizumab as initial therapy for metastatic colorectal cancer. Retrospective analysis of the TRIBE trial by GONO. Ann Oncol. (2018) 29:152834. doi: 10.1093/annonc/mdy140

16. Hegewisch-Becker S, Nöpel-Dünnebacke S, Hinke A, Graeven U, ReinacherSchick A, Hertel J, et al. Impact of primary tumour location and RAS/BRAF mutational status in metastatic colorectal cancer treated with first-line regimens containing oxaliplatin and bevacizumab: prognostic factors from the AIO KRK0207 first-line and maintenance therapy trial. Eur J Cancer. (2018) 101:105-13. doi: 10.1016/j.ejca.2018.06.015

17. Jordan F, Grundmann N, Schenkirsch G, Märkl B, Messmann H, Anthuber $\mathrm{M}$, et al. Impact of primary tumor localization on the efficacy of bevacizumab in metastatic colorectal cancer. Anticancer Res. (2018) 38:553946. doi: 10.21873/anticanres.12889

18. Snyder $M$, Bottiglieri $S$, Almhanna K. Impact of primary tumor location on first-line bevacizumab or cetuximab in metastatic colorectal cancer. Rev Recent Clin Trials. (2018) 13:139-49. doi: 10.2174/1574887113666180328104109

19. Loupakis F, Yang D, Yau L, Feng S, Cremolini C, Zhang W, et al. Primary tumor location as a prognostic factor in metastatic colorectal cancer. J Natl Cancer Inst. (2015) 24:dju427. doi: 10.1093/jnci/djv207

20. Aljehani MA, Morgan JW, Guthrie LA, Jabo B, Ramadan M, Bahjri K, et al. Association of primary tumor site with mortality in patients receiving bevacizumab and cetuximab for metastatic colorectal cancer. JAMA Surg. (2018) 153:60-7. doi: 10.1001/jamasurg.2017.3466

21. Calvetti L, Pavarana M, Auriemma A, Tondulli L, Bria E, Molino A, et al. Effectiveness of Cetuximab (Cet) and Bevacizumab (Bev) for metastatic colorectal cancer (mCRC) according to primary tumor location (PTL): findings from a 'real-world' retrospective analysis. Ann Oncol. (2015) 26:vi401. doi: $10.1093 /$ annonc/mdv340.15 
22. Lu HJ, Lin JK, Chen WS, Jiang JK, Yang SH, Lan YT, et al. Primary tumor location is an important predictive factor for wild-type KRAS metastatic colon cancer treated with cetuximab as front-line bio-therapy. Asia Pac J Clin Oncol. (2016) 12:207-15. doi: 10.1111/ajco.12469

23. He WZ, Liao FX, Jiang C, Kong PF, Yin CX, Yang Q, et al. Primary tumor location as a predictive factor for first-line bevacizumab effectiveness in metastatic colorectal cancer patients. J Cancer. (2017) 8:388-94. doi: 10.7150/jca.16804

24. Sun DC, Shi Y, Wang YR, Lv Y, Yan H, Mao H, et al. KRAS mutation and primary tumor location do not affect efficacy of bevacizumab-containing chemotherapy in stagae IV colorectal cancer patients. Sci Rep. (2017) 7:14368. doi: 10.1038/s41598-017-14669-2

25. Houts AC, Ogale S, Sommer N, Satram-Hoang S, Walker MS. Treatment patterns and outcomes in patients with KRAS wild-type metastatic colorectal cancer treated in first line with bevacizumabor cetuximab-containing regimens. J Gastrointest Cancer. (2019) 50:69-77. doi: 10.1007/s12029-017-0027-6

26. Bazarbashi S, Omar A, Aljubran AH, Alzahrani AM. Response rate and survival for patients with metastatic colorectal cancer from right-sided versus left-sided tumors, treated with first-line triplet chemotherapy with bevacizumab. J Clin Oncol. (2017) 35:801. doi: 10.1200/JCO.2017.35.4_suppl.801

27. Ulivi P, Scarpi E, Chiadini E, Marisi G, Valgiusti M, Capelli L, et al. Rightvs. left-sided metastatic colorectal cancer: differences in tumor biology and bevacizumab efficacy. Int J Mol Sci. (2017) 18:1240. doi: 10.3390/ijms18061240

28. Arora SP, Ketchum NS, Michalek J, Gelfond J, Mahalingam D. Left versus right: does location matter for refractory metastatic colorectal cancer patients in phase 1 clinical trials? J Gastrointest Cancer. (2018) 49:2837. doi: 10.1007/s12029-017-9948-3

29. Demircan N, Dane F, Ozturk MA, Besiroglu M, Babacan N, Kays S, et al. Analysis of survival and prognostic factors in metastatic colorectal cancer patients treated with first line bevacizumab. J Clin Oncol. (2017) 35:e15009. doi: 10.1200/JCO.2017.35.15_suppl.e15009

30. Reinacher-Schick AC, Noepel-Duennebacke S, Hertel J, Tannapfel A, Arnold D, Hinke A, et al. Localization of the primary tumor (LPT) and maintenance strategies after first line oxaliplatin (Ox), fluoropyrimidine (FP), and bevacizumab (Bev) in metastatic colorectal cancer (mCRC): results from the AIO 0207 trial. J Clin Oncol. (2017) 35:3543. doi: 10.1200/JCO.2017.35.15_suppl.3543

31. Artaç M, Korkmaz L, Coşkun HS, Dane F, Karabulut B, Karaagaç $M$, et al. Bevacuzimab may be less effective in obese metastatic colorectal cancer patients. J Gastrointest Cancer. (2018) 50:214-22. doi: 10.1007/s12029-017-0047-2

32. Loupakis F, Hurwitz HI, Saltz L, Arnold D. Efficacy outcomes with bevacizumab added to chemotherapy (bev+CT) compared with chemotherapy alone (CT) in left- and rightsided tumors in metastatic colorectal cancer (mCRC). J Clin Oncol. (2018) 36:726. doi: 10.1200/JCO.2018.36.4_suppl.726

33. Satake H, Sunakawa Y, Miyamoto Y, Nakamura M, Nakayama H, Shiozawa $\mathrm{M}$, et al. A phase II trial of 1st-line modified-FOLFOXIRI plus bevacizumab treatment for metastatic colorectal cancer harboring RAS mutation: JACCRO CC-11. Oncotarget. (2018) 9:18811-20. doi: 10.18632/oncotarget. 24702

34. Chibaudel B, Andre T, Samson B, Garcia-Larnicol M-L, Dauba J, Lledo $G$, et al. Impact of primary tumor sidedness on erlotinib efficacy in patients with metastatic colorectal cancer treated with bevacizumab maintenance: results from the DREAM phase III trial. J Clin Oncol. (2018) 36:737. doi: 10.1200/JCO.2018.36.4_suppl.737

35. Nakamura $M$, et al. Prognostic impact of tumor location and use of monoclonal antibodies in patients with metastatic colorectal cancer. Ann Oncol. (2016) 27:ix58. doi: 10.1093/annonc/mdw581.018

36. de Andrea CE, Schalper KA, Sanmamed MF, Melero I.de Andrea CE. Immunodivergence in metastatic colorectal cancer. Cancer Cell. (2018) 34:876-8. doi: 10.1016/j.ccell.2018.11.012

37. Brody H. Colorectal cancer. Nature. (2015) 521:S1. doi: 10.1038/521S1a
38. Modest DP, Pant S, Sartore-Bianchi A. Treatment sequencing in metastatic colorectal cancer. Eur J Cancer. (2019) 109:70-83. doi: 10.1016/j.ejca.2018.12.019

39. New guideline on managing colorectal cancer. Cancer Discov. (2017) 7:OF2. doi: 10.1158/2159-8290.CD-NB2017-030

40. Kather JN, Halama N, Jaeger D. Genomics and emerging biomarkers for immunotherapy of colorectal cancer. Semin Cancer Biol. (2018) 52(Pt 2):18997. doi: 10.1016/j.semcancer.2018.02.010

41. Loupakis F, Hurwitz HI, Saltz L, Arnold D, Grothey A, Nguyen QL, et al. Impact of primary tumour location on efficacy of bevacizumab plus chemotherapy in metastatic colorectal cancer. Br J Cancer. (2018) 119:14515. doi: 10.1038/s41416-018-0304-6

42. Yang J, Du XL, Li ST, Wang BY, Wu YY, Chen ZL, et al. Characteristics of differently located colorectal cancers support proximal and distal classification: a population-based study of 57,847 patients. PLoS ONE. (2016) 11:e0167540. doi: 10.1371/journal.pone.0167540

43. Chen QG, Zhang L, Sun F, Li SQ, You XH, Jiang YH, et al. Elevated FPR confers to radiochemoresistance and predicts clinical efficacy and outcome of metastatic colorectal cancer patients. Aging. (2019) 11:171632. doi: 10.18632/aging.101864

44. Bendardaf R, Buhmeida A, Hilska M, Laato M, Syrjänen S, Syrjänen K, et al. VEGF-1 expression in colorectal cancer is associated with disease localization, stage, and long-term disease-specific survival. Anticancer Res. (2008) 28:386570. doi: 10.3109/07357900802672761

45. Hutajulu SH, Paramita DK, Santoso J, Sani MIA, Amalia A, Wulandari G, et al. Correlation between vascular endothelial growth factor-A expression and tumor location and invasion in patients with colorectal cancer. J Gastrointest Oncol. (2018) 9:1099-108. doi: 10.21037/jgo.2018.07.01

46. Kim K, Castro EJT, Shim H, Advincula JVG, Kim YW. Differences regarding the molecular features and gut microbiota between right and left colon cancer. Ann Coloproctol. (2018) 34:280-5. doi: 10.3393/ac.2018.12.17

47. Smeets D, Miller IS, O'Connor DP, Das S, Moran B, Boeckx B, et al. Copy number load predicts outcome of metastatic colorectal cancer patients receiving bevacizumab combination therapy. Nat Comm. (2018) 9:4112. doi: 10.1038/s41467-018-06567-6

48. Guinney J, Dienstmann R, Wang X, de Reyniès A, Schlicker A, Soneson $\mathrm{C}$, et al. The consensus molecular subtypes of colorectal cancer. Nat Med. (2015) 21:1350-6. doi: 10.1038/nm.3967

49. Lee MS, Menter DG, Kopetz S. Right versus left colon cancer biology: integrating the consensus molecular subtypes. J Natl Compr Canc Netw. (2017) 15:411-9. doi: 10.6004/jnccn.2017.0038

50. Gao Z, Guo B, Gao R, Zhu Q, Qin H. Microbiota disbiosis is associated with colorectal cancer. Front Microbiol. (2015) 6:20. doi: 10.3389/fmicb.2015.00020

51. Coker OO, Nakatsu G, Dai RZ, Wu WKK, Wong SH, Ng SC, et al. Enteric fungal microbiota dysbiosis and ecological alterations in colorectal cancer. Gut. (2018) 68:654-62. doi: 10.1136/gutjnl-2018-317178

52. Gopalakrishnan V, Spencer CN, Nezi L, Reuben A, Andrews MC, Karpinets TV, et al. Gut microbiome modulates response to antiPD-1 immunotherapy in melanoma patients. Science. (2018) 359:97103. doi: 10.1126/science.aan4236

53. Yu T, Guo F, Yu Y, Sun T, Ma D, Han J, et al. Fusobacterium nucleatum promotes chemoresistance to colorectal cancer by modulating autophagy. Cell. (2017) 170:548-63 e16. doi: 10.1016/j.cell.2017.07.008

Conflict of Interest Statement: The authors declare that the research was conducted in the absence of any commercial or financial relationships that could be construed as a potential conflict of interest.

Copyright (c) 2019 You, Wen, Xia, Sun, Li, Wang, Fang, Chen, Zhang, Jiang, Wang, Ying and Zong. This is an open-access article distributed under the terms of the Creative Commons Attribution License (CC BY). The use, distribution or reproduction in other forums is permitted, provided the original author $(s)$ and the copyright owner(s) are credited and that the original publication in this journal is cited, in accordance with accepted academic practice. No use, distribution or reproduction is permitted which does not comply with these terms. 